\title{
Thermal Desorption in Pure Hexane and Hexane/Butane Mixtures on Graphite
}

\author{
Cary L. Pint \\ Department of Physics \\ University of Northern lowa \\ Cedar Falls, lowa 50614-0150 USA
}

Received:November 19, 2004

Accepted: November 29, 2004

\begin{abstract}
The results of an extensive study of desorption in hexane (monolayer and submonolayer) and hexane/butane mixtures initially adsorbed onto the graphite basal plane is presented. Molecular dynamics (MD) simulations are utilized to carry out atomistic simulations at temperatures $300 \leq T \leq 700$ for all three cases studied. Results from submonolayer and monolayer hexane indicate that the desorption energy needed for the system to proceed with the desorption process is independent of the system's coverage, which is in good agreement with experimental results. It is also found that simulations of desorption of hexane/butane mixtures yield a high ratio of butane molecules being desorbed from the surface at lower temperatures $(300 \mathrm{~K})$, suggesting a strong dependence of the desorption barrier upon alkane chain length. The results are discussed and compared to previous experimental and theoretical results.
\end{abstract}

\section{INTRODUCTION}

The study of the properties of the phases and phase transitions of $n$-alkanes on surfaces has been a rich topic of experimental and theoretical interest recently due to advancements in methods to study these systems on a microscopic scale (scanning tunneling microscopy and other probing techniques) as well as increasing ability of computers to simulate models with large enough system sizes to accurately represent these types of systems. Namely, the family of $n$-alkanes is of particular interest due to the vast amount of applications these molecules provide to industry (lubrication, adhesion, wetting on surfaces, etc).

In the past 30 years, considerable progress has been made in understanding the dynamics of $n$-alkane adsorption onto various types of surfaces. Although, despite this progress, the studies of $n$-alkane desorption from surfaces is still a topic that is not understood reasonably well, and poses many theoretical questions.

The two molecules that are studied in this work are primarily hexane $\left(\mathrm{C}_{6} \mathrm{H}_{14}\right)$ and also butane $\left(\mathrm{C}_{4} \mathrm{H}_{10}\right)$. Both of these molecules are members of the family of straight-chained $n$-alkanes, which is a family of molecules that differ primarily in their length. Butane and hexane are specifically chosen due to their balance of intermediate size and many degrees of freedom they display as compared to other complex organic molecules. For this reason, these molecules have been studied extensively as adsorbates.

The surface that is simulated in this case is graphite. Graphite is arguably the best candidate for a substrate due to its symmetric nature, vast availability, and strong mechanical stability. These qualities, as well as the large amount of experimental and theoretical work that exists for systems involving graphite makes it an excellent choice as a substrate in such a simulation. 
There have been many previous experimental [1-3] and theoretical [4-11] studies of hexane and butane on graphite. In the studies of hexane, a solid commensurate herringbone structure is observed at low temperatures, followed by a transition into an intermediate orientationally ordered nematic phase, which is then followed by a transition into an isotropic fluid. In all cases, the phase transitions studied are found to be dependent upon the system's in-plane room (e.g. the intermediate nematic phase is found to be strictly a result of effects resulting from inplane molecular packing [12]). Similarly, previous work that studies butane on graphite [2,4-5,9] finds a commensurate herringbone formation at low temperatures followed by an abrupt melting transition into an isotropic fluid.

There have also been many broad experimental and theoretical studies of desorption of $n$-alkanes on graphite. The first experimental study of desorption of hydrocarbons on surfaces was conducted by Zhang and Gellman [13] over a series of straight chain alcohols on the $\mathrm{Ag}(110)$ surface. This work finds that the desorption energy increases incrementally by $4.6 \pm 0.4$ $\mathrm{kJ} /$ mole per methylene group in the hydrocarbon chain. More recently, experimental work has been conducted by Paserba and Gellman [14, 15] that deals with desorption over a series of $n$-alkanes. The two major conclusions of this work are (i) the desorption energy is independent of the surface coverage, and (ii) the desorption energies increase nonlinearly with increasing chain length due to less molecules in the trans configuration.

The theoretical work that has been done on desorption of the $n$-alkanes has been completed by Fichthorn et al [16-18]. In these studies, a molecular dynamics simulation of a single molecule on a $\mathrm{Pt}(111)$ surface is carried out for various molecules of differing chain length. These studies find both a large increase of molecules out of their trans conformations at longer chain lengths that contribute to a nonlinear effect on the desorption energy, and also find that calculations of desorption rates using transition-state theory (TST) are in correspondence with experiment.

The purpose of this work is to (i) study desorption of hexane on graphite through use of two differing densities (submonolayer and monolayer) to confirm the experimental result that the desorption barrier is independent of coverage; (ii) study the effects on the desorption process for $n$ alkane mixtures which is currently both theoretically and experimentally unexplored; and (iii) study the desorption process in these systems through use of larger system sizes than were considered in previous theoretical work.

\section{COMPUTATIONAL DETAILS}

This study is conducted through use of a constant particle number, planar density, and temperature $(N, \rho, T)$ molecular dynamics (MD) method. To model the hexane and butane molecules, a united atom (UA) model is utilized which effectively approximates each methyl $\left(\mathrm{CH}_{3}\right)$ and methylene $\left(\mathrm{CH}_{2}\right)$ group as a single pseudoatom with an increased Van der Waals radius from that of a single carbon atom. This model reduces the number of force sites that are included in calculations of forces and neighbor lists within the simulation, which tend to be very time intensive (e.g. the neighbor list calculations scale as $N^{2}$, where $N$ is the total number of psuedo-atoms in the simulation). In all simulations of hexane, $N=672$, which corresponds to 112 hexane molecules, and similarly in simulations of hexane/butane mixtures, $N=560$, which corresponds to 56 butane molecules and 56 hexane molecules. The two densities that are studied for hexane correspond to $\rho=1$ (monolayer completion) and $\rho=0.903$ (submonolayer). In the case of the submonolayer density studied, the computational cell is uniformly expanded in the $y$ direction by a factor of $n a_{g}$, where $a_{g}$ is the graphite lattice constant that takes on a value of $a_{g}=2.46 \AA$ and $n=$ 3 for this specific case. The cell size used in simulations of monolayer hexane is $a=$ $68.1735 \AA$ and $b=68.88 \AA$, and in the case of submonolayer hexane, $a=68.1735 \AA$ and $b=76.26 \AA$, where $a$ and $b$ correspond to orthogonal directions parallel to the plane of the substrate. For simulations of hexane/butane mixtures, the cell size is $a=$ $67 \AA$ and $b=67.1 \AA$. Periodic boundary conditions are utilized in the $a$ and $b$ directions, and free boundary conditions are implemented in the $z$ direction. To simulate 
the system at constant temperature, the velocities are frequently rescaled to satisfy equipartition for the center-of-mass, rotational, and internal temperatures,

$$
\begin{gathered}
T_{C M}=\frac{1}{3 N_{m} k_{B}} \sum_{i=1}^{N_{m}} M_{i} v_{i, C M}^{2}, \\
T_{R O T}=\frac{1}{3 N_{m} k_{B}} \sum_{i=1}^{N_{m}} \vec{\omega}_{i}{ }^{T} \vec{I}_{i} \vec{\omega}_{i}, \\
T_{I N T}=\frac{1}{2 n_{C}-5} \sum_{i=1}^{N_{m}} \sum_{j=1}^{n_{C}} m_{i j}\left(\vec{v}_{i j}-\vec{v}_{i, C M}-\vec{\omega}_{i, C M} \times \vec{r}_{i j, C M}\right)^{2} .
\end{gathered}
$$

where $T_{C M}, T_{R O T}$ and $T_{I N T}$ are the temperatures of the system and the summation index (i) runs over molecules wile the index (j) runs over pseudo-atoms within a given molecule. All variables indexed with (i) are standard and apply to the $i^{\text {th }}$ molecule and those indexed with (ij) apply to the $f^{\text {th }}$ atom within molecule (i).

A velocity Verlet RATTLE algorithm, with a time step of $1 \mathrm{fs}$, carries out the integration of the equations of motion. In all cases, the system is initially started from a solid herringbone configuration that is experimentally verified. The system is then allowed to equilibrate for $8 \times 10^{3}$ steps, which is found to be sufficient time to disrupt the solid configuration of the system into a fluid and to keep the desorbate under the influence of the substrate interaction. A period of $3 \times 10^{5}$ steps (300 ps) then follows where the behavior of the system is studied and various useful averages and distributions are calculated. The system is progressed through a temperature sequence of $\mathrm{T}=300 \mathrm{~K}$ $+n(10 \mathrm{~K})$, where $n=1,2,3, \ldots$ and $0 \leq n \leq$ 40 for all three cases studied.

To model the potential interactions, both non-bonded and bonded interactions are used. The first of the non-bonded interactions is the molecule-molecule interaction, modeled by the Lennard Jones pair potential

$$
u_{L J}\left(r_{i j}\right)=4 \varepsilon_{i j}\left[\left(\frac{\sigma_{i j}}{r_{i j}}\right)^{12}-\left(\frac{\sigma_{i j}}{r_{i j}}\right)^{6}\right] .
$$

In equation (1), the Lorentz-Berthelot combining rules

$$
\begin{gathered}
\sigma_{i j}=\frac{\sigma_{i}+\sigma_{j}}{2} \\
\varepsilon_{i j}=\sqrt{\varepsilon_{i} \varepsilon_{j}}
\end{gathered}
$$

are applied in order to describe mixed interactions when particles (i) and (j) are of different types.

The other non-bonded interaction representing the molecule-substrate interaction is given by a Fourier expansion proposed by W.A. Steele [15], and is of the form:

$$
u_{i}^{g r}=E_{0 i}\left(z_{i}\right)+\sum_{n=1}^{\infty} E_{n i}\left(z_{i}\right) f_{n i}\left(x_{i}, y_{i}\right),
$$

For information on the parameters and quantities shown in equation (2), the reader is referred to [11].

For this study, there are two types of bonded interactions involved as well. The first bonded interaction is bond angle bending. This is a three-body interaction, with the bending potential defined as [16],

$$
u_{\text {bend }}=k_{\theta}\left(\theta_{b}-\theta_{0}\right)^{2},
$$

where $\theta_{b}$ is the bond angle, $\theta_{0}$ is the equilibrium bond angle and $k$ is the angular stiffness. Similarly, the other bonded interaction that is considered is the dihedral (torsional) [7,17] bending, and the bending potential is defined as: 


$$
u_{\text {tors }}=\sum_{i=0}^{5} c_{i}\left(\cos \phi_{d}\right)^{i},
$$

where $\phi_{d}$ is the dihedral angle and the $c_{i}$ are constants. For values of parameters used in Equations (3) and (4), the reader is again referred to [11]. For this study, the use of the RATTLE algorithm [18] provides a constrained solution to the equations of motion to keep the bond lengths fixed at $1.54 \AA$.

\section{RESULTS}

Many runs were carried out over the temperature region of $300 \mathrm{~K} \leq T \leq 700 \mathrm{~K}$ to attempt to classify the characteristics of the system as the temperature is increased, but also to repress the large statistical error that is dealt with when dealing with desorption in smaller systems. In fact, the process of desorption takes place over such large time scales, it is impossible to be completely probed through an MD simulation. Through this section, various quantities will be presented to provide a clear picture of the desorption process that occurs in both monolayer and submonolayer hexane and compare that to the desorption process observed in mixtures of hexane and butane.

The first quantity that is of interest is the tilt angle order parameter. This order parameter is defined as

$$
\text { OPtilt }=\frac{1}{2 N_{m}}\left\langle\sum_{i=1}^{N_{m}}\left(3 \cos ^{2} \theta_{i}-1\right)\right\rangle,
$$

and is the thermal average of a Legendre polynomial $\left(P_{2}\right)$ and takes a value of -0.5 if the long axis of each hexane molecule is parallel to the $(x, y)$ plane. This quantity gives information on the libration of the molecules out of the surface plane parallel to the graphite substrate. In the process of desorption, large values of OPtilt are observed due to the large thermal energies that are present at the higher temperatures that are needed to conduct simulations of desorption. The temperature dependence of OPtilt is shown in Figure 1 for submonolayer and monolayer hexane.

Another useful way to analyze the behavior of the system is to study the behavior of the system's energies. Probably the most significant of these are the

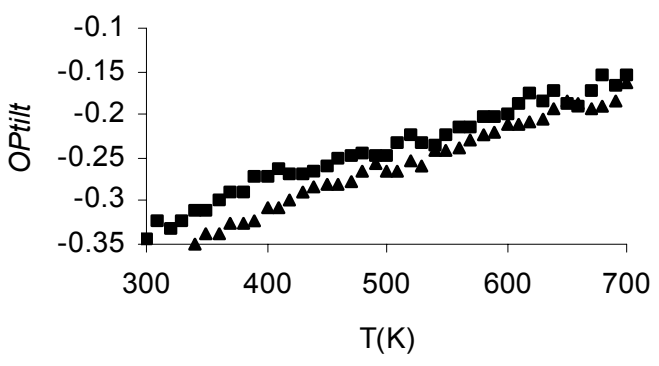

Figure 1. Temperature dependence of OPtilt for monolayer (solid squares) and submono-layer (solid triangles) hexane.

energies that describe the moleculemolecule and the molecule-substrate interaction. The first of these (the moleculemolecule energy) is given by the Lennard Jones pair potential function:

$$
<U_{L J}>=\frac{1}{N_{m}}\left\langle\sum_{i=1}^{N} \sum_{j=i+1}^{N} u_{L J}\left(r_{i j}\right)\right\rangle
$$

and is useful when considering the system's structural behavior independent of the graphite substrate. The other is the average lateral Steele corrugation energy per molecule, given by:

$$
<U_{1}>=\frac{1}{N_{m}}\left\langle\sum_{i=1}^{N} E_{n i}\left(\vec{r}_{i}\right)\right\rangle
$$

where the value of $\left\langle U_{1}\right\rangle$ vanishes when molecules are randomly sampling positions in the $(x, y)$ plane, and is significant when the molecules are strongly bound to the graphite surface. The temperature dependence of both of these energies is shown in Figure 2 for the cases of submonolayer and monolayer hexane.

Although these quantities tell very important information, they give little information regarding the process of desorption. Therefore, the calculations that were conducted regarding desorption are shown in Figures 4-6. Also, in Figure 3, the desorption process is illustrated through a series of snapshots at progressing times throughout the simulation at $T=500 \mathrm{~K}$. 

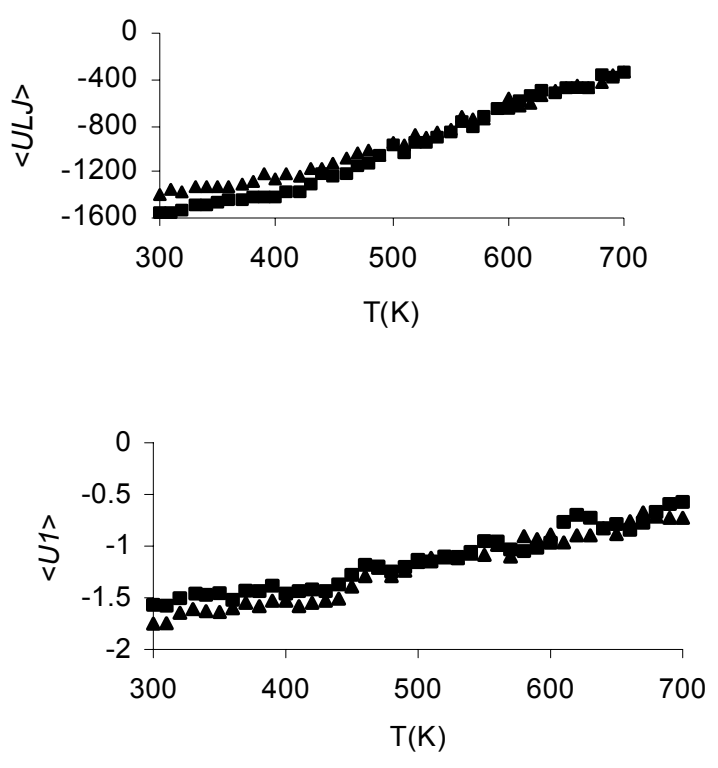

Figure 2. Average Lennard Jones (top) and average corrugation energies as a function of temperature. Again, the solid squares correspond to monolayer hexane, and the solid triangles correspond to submonolayer hexane.

The number of desorbed molecules was monitored throughout the simulation at every 100 time steps ( $0.1 \mathrm{ps}$ ) to classify the desorption activity as a function of time. In Figure 4, plots of desorbed molecules as a function of time are shown for various temperatures for the three cases of butane/hexane mixtures, submonolayer hexane, and monolayer hexane. These graphs show significant desorption activity throughout the first portion of the simulation, with the activity becoming less significant as time evolves. Similarly, in Figure 5a, the temperature dependence of the total number of desorbed molecules is presented for each of the three cases studied, with the separate cases of desorption for butane and hexane in the butane/hexane mixture as a function of temperature shown in Figure $5 \mathrm{~b}$.

Another useful way to characterize the desorption in the system is to analyze the diffusion coefficient of the molecules. Although one would consider using the useful Einstein relation to calculate diffusion coefficients, due to periodic boundary conditions, the rms displacement formula would not give an accurate representation of this quantity. Therefore, the velocity autocorrelation function is used in the relation for the three-dimensional diffusion coefficient:

$$
D=\frac{1}{3} \int_{0}^{\infty} v(t) v(0) d t
$$

where $v(t)$ and $v(0)$ are the velocity autocorrelations at time $t$ and $t_{0}$ respectively. The temperature dependence of $D$ is shown in Figure 6 for all three cases studied. The trend that is evident in these plots is generally the same trend that is evident from the plots in Figure 5, with exception of the diffusion coefficients for butane/hexane mixtures, where the temperature dependence of $D$ seems to be a little more nonlinear than is the case for pure hexane at submonolayer and monolayer coverages.

\section{DISCUSSION}

This section will be split into two subsections. The first subsection will deal with comparisons between results of submonolayer and monolayer hexane, and will compare how the desorption rate changes as the thermal energy is increased in each of these systems, and if the results correspond to experimental work. The second subsection will focus on the behavior of butane/hexane mixtures compared to pure hexane, and will comment on the similarities and differences of the two systems to study the effects of desorption in mixtures where there are two molecules of differing chain length present.

\section{a. Monolayer and Submonolayer Hexane}

In adsorbed systems of monolayer and submonolayer hexane that have been studied previously, the effects of in-plane room upon the phase transitions brought about a considerably noticeable effect at lower temperatures. In this case, the differences between these two systems as they desorb is very minimal. In Figure 1 , it is clear that the values of OPtilt for the monolayer case are a little greater than the values of OPtilt for the submonolayer case. This is consistent with previous work monolayer $[11,12]$ conducted on 

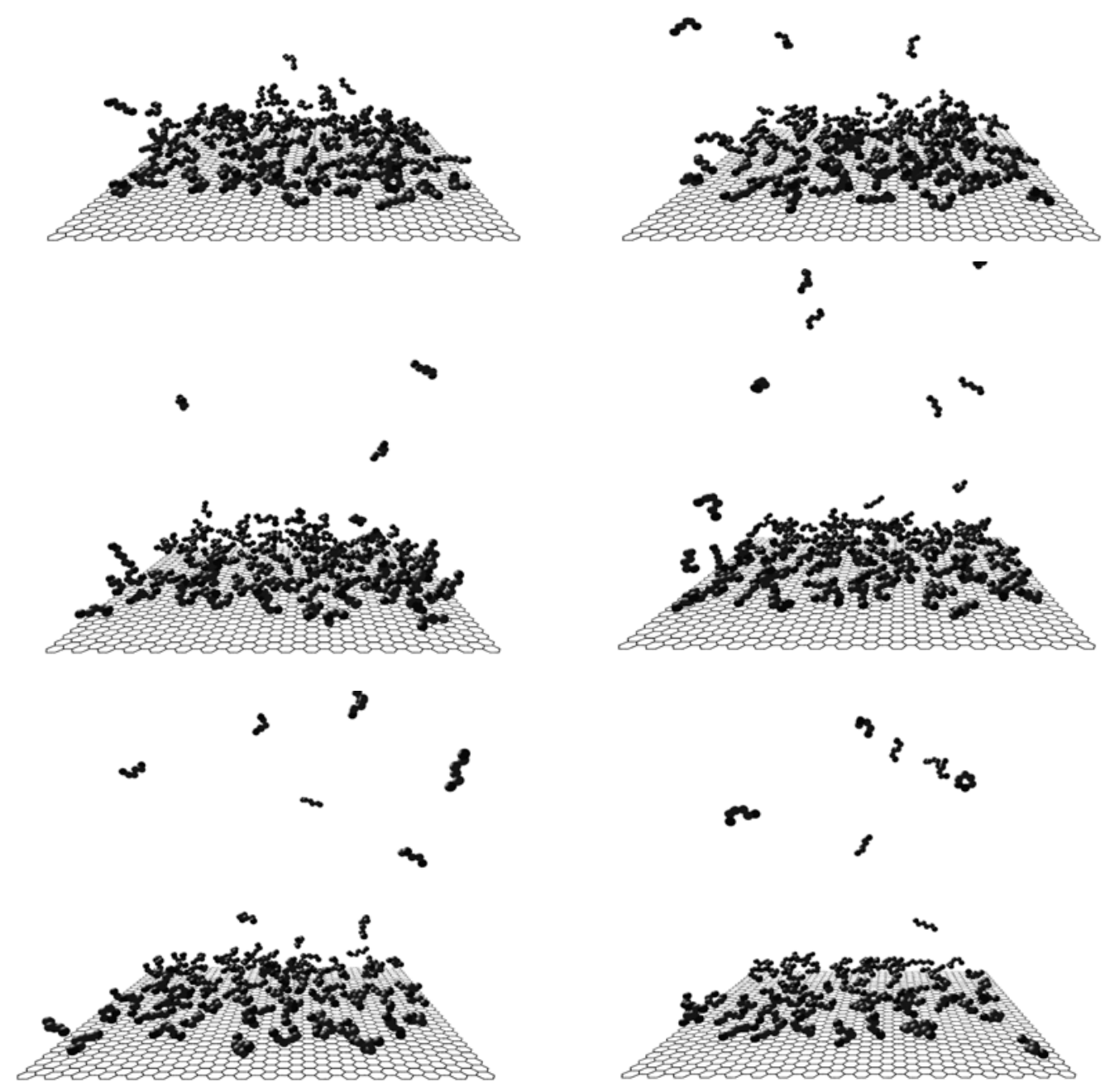

Figure 3. Snapshots of system configurations for monolayer hexane at $T=600 \mathrm{~K}$ at (starting from top left and moving left to right). Top: 0.1 ps., 5 ps. Middle: 10 ps., 50 ps. Bottom: 150 ps., 250 ps. Each 1 ps. is 1000 simulation time steps.

submonolayer and hexane at lower temperatures. However, in Figure 2, the average Lennard Jones and corrugation potentials are very nearly the same, with the same general decreasing trend observed in each. Further, Figures 4-6 show that the diffusion rates of the molecules in both cases are very nearly identical. In Figure 4, the time dependence of the desorption in both cases is very similar. This is also evident in Figure 5, where the desorption barrier (the temperature at which the molecules have enough energy to desorb from the surface) is at about $410-420 \mathrm{~K}$ in both cases, and increases linearly in the same fashion with increasing temperature. Also, the behavior of the diffusion coefficients of these two systems seems to be very similar, within reasonable statistical error.

These results are strong evidence that the desorption barrier in this system of hexane on graphite, is generally 

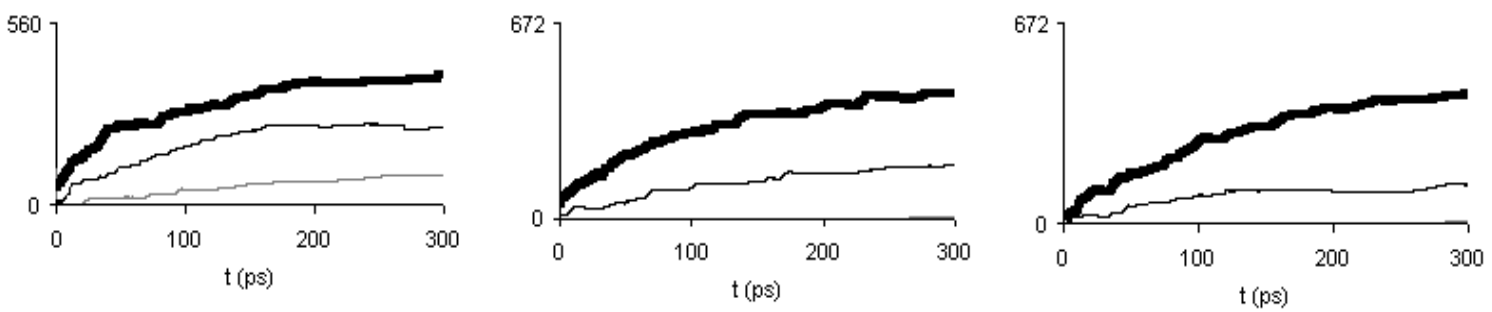

Figure 4. Time dependence on the desorption process per pseudo-atom for butane/hexane mixtures (left), monolayer hexane (middle), and submonolayer hexane (right). The three lines represent three different temperature points: $T=400 \mathrm{~K}$ (gray), $T=550 \mathrm{~K}$ (black), and $T=700 \mathrm{~K}$ (thick black). The vertical axis on each plot represents the total number of pseudo-atoms included in the simulation.

independent of coverage. Therefore, this means that the energy needed for the molecules to desorb from the surface does not depend on coverage, but rather temperature. This result is indication of a first order process, and has been experimentally labeled as such [14-15].

\section{b. Butane/Hexane Mixtures}

In contrast to the study of monolayer and submonolayer hexane, the study of the mixture of butane and hexane molecules introduces the dependence of the desorption barrier upon chain length that has previously been observed in all experimental work ${ }^{13-15}$. This is clear when considering the temperature dependence of the number of desorbed molecules after 300 ps, as shown in Figure 5a. This suggests that the desorption barrier for hexane/butane mixtures is at $\sim 300 \mathrm{~K}$, which is much lower than the $410-420 \mathrm{~K}$ as proposed for hexane.

Further, from Figure 5a, it seems as if the desorption process is linear with increasing temperature after the desorption barrier is reached for both cases of hexane. However, Figure $5 \mathrm{~b}$ suggests that in a butane/hexane mixture, the desorption process for each species as a function of temperature is not linear. Although, interestingly, when considering the total number of pseudo-atoms desorbed after a period of 300 ps in the mixture, the result (the sum of the two individual plots for butane and hexane in Figure $5 \mathrm{~b}$ ) is seemingly linear with increasing temperature.
This behavior suggests that in this mixture, the desorption process is cooperative between butane and hexane


Figure 5a. Temperature dependence of desorbed molecules for butane/hexane mixtures (top), monolayer hexane (middle), and submonolayer hexane (bottom). 
Butane

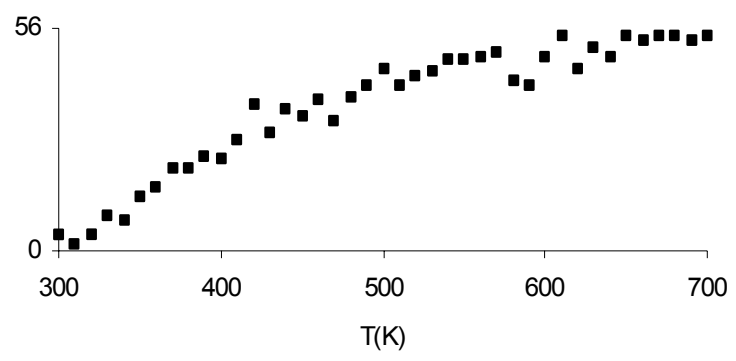

Hexane

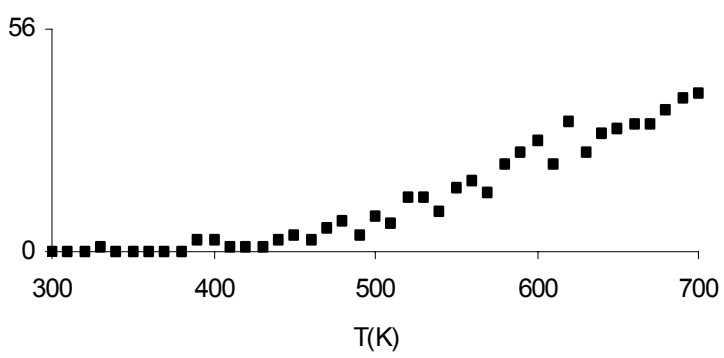

Figure 5b. Temperature dependence on the desorption of butane (left) and hexane (right) in simulated butane/hexane mixtures. In contrast to $5 \mathrm{a}$, the vertical axis both of plots shown here indicate the total number of molecules of each type considered (butane and hexane).

molecules. This means that regardless of the fluctuations in the desorption of butane or hexane molecules specifically within the mixture, the net effect of desorption of molecules in the system as a function of temperature is linear, and the therefore the molecules within the system cooperate to produce this temperature-driven linear desorption effect.

\section{a. Other Comments}

It should be mentioned that error bars were not used in any plots to give indication of statistical error present due to the large number of temperature points that were run and plotted. Also, in all cases of butane/hexane mixtures, the system had the same qualitative behavior for OPtilt, $\left\langle U_{1}\right\rangle$, and $\left\langle U_{L J}\right\rangle$ as was observed for hexane, so the author found it unnecessary to include plots of these.

\section{CONCLUSIONS}

The conclusions of this work are (i) desorption in the system of hexane on graphite is independent of coverage, and therefore exhibits first-order like transition properties, and (ii) desorption in systems of hexane on graphite and butane/hexane mixtures on graphite produce a linear trend in desorption as a function of temperature. In the case of butane/hexane mixtures, this trend is non-linear for each individual species, but the net effect is still seemingly linear, which is evidence that the desorption
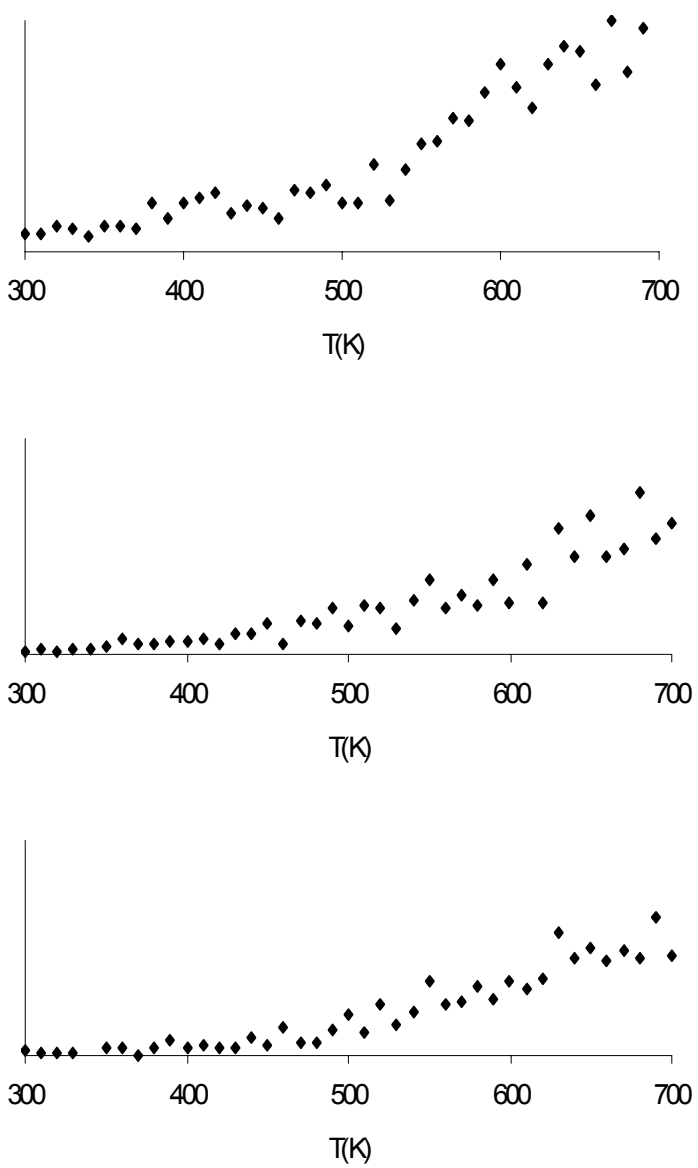

Figure 6. Temperature dependence of the three-dimensional diffusion coefficient for butane/hexane mixtures (top), monolayer hexane (middle), and submonolayer hexane (bottom). The numerical value on the vertical axis is identical and arbitrary for all plots shown. 
process is cooperative between species in mixtures of alkanes of different chain length.

\section{ACKNOWLEDGEMENTS}

The author is indebted to Dr. Paul Gray and the UNI Computer Science department for invaluable use of CPU time that allowed this research to be carried out. Also, a special thanks is extended to Dr. Michael Roth for continuous support in all current projects and ideas the author is currently working on.

\section{REFERENCES}

1. J. Krim, J. Suzanne and H. Shechter, R. Wang and H. Taub, Surf. Sci. 162 (1-3) 446 (1985).

2. Newton, dissertation under $\mathrm{H}$. Taub, unpublished.

3. H. Taub, Vol. 228 of NATO Advanced Study Institutes, Series C: Mathematical and Physical Sciences, edited by G. J. Long and F. Grandjean (Kluwer, Dordrecht, 1988), pp. 467-497.

4. Flemming $\mathrm{Y}$. Hansen and $\mathrm{H}$. Taub, Phys. Rev. Lett., 69 (4), 652 (1992).

5. Flemmimg Y. Hansen, J.C. Newton and H. Taub, J. Chem. Phys., 98 (5), 4128 (1993).

6. E. Velasco and Gunther H. Peters, J. Chem. Phys., 102 (2) 1098 (1995).
7. G.H. Peters and D.J. Tildesley, Langmuir 121557 (1996).

8. Gunther H. Peters, Surf. Sci., 347169 (1996).

9. K. W. Herwig, Z. Wu, P. Dai, H. Taub, and F. Y. Hansen, J. Chem. Phys. 107, 5186-5196 (1997).

10. M. Krishnan and S. Balasubramanian, S. Clarke, J. Chem. Phys., 118 (11) 5082 (2003)

11. M.W. Roth, C.L. Pint, and Carlos Wexler, Phys. Rev. B, (submitted)

12. Cary Pint and M.W. Roth, (to be submitted)

13. R. Zhang and A. Gellman, J. Phys. Chem., 95, 7433 (1991)

14. K. Paserba and A. Gellman, Phys. Rev. Lett., 86, 4338 (2001)

15. K. Paserba and A. Gellman, J. Chem. Phys., 115, 6737 (2001)

16. D. Huang, Y. Chen, and K. A. Fichthorn, J. Chem. Phys, 101, 11021 (1994)

17. J.S. Raut and K. A. Fichthorn, J. Chem. Phys., 108, 1626 (1999)

18. K. A. Fichthorn and R.A. Miron, Phys. Rev. Lett., 89, 196103 (2002)

19. W.A. Steele, Surf. Sci. 36, 317 (1973).

20. Marcus G. Martin and J. Ilja Siepmann, J. Phys. Chem., 102, 2569 (1998)

21. P.Padilla and S. Toxværd, J. Chem. Phys. 945650 (1991).

22. M. P. Allen, D. J. Tildesley, Computer simulation of liquids, Clarendon Press, New York, NY, 1988.
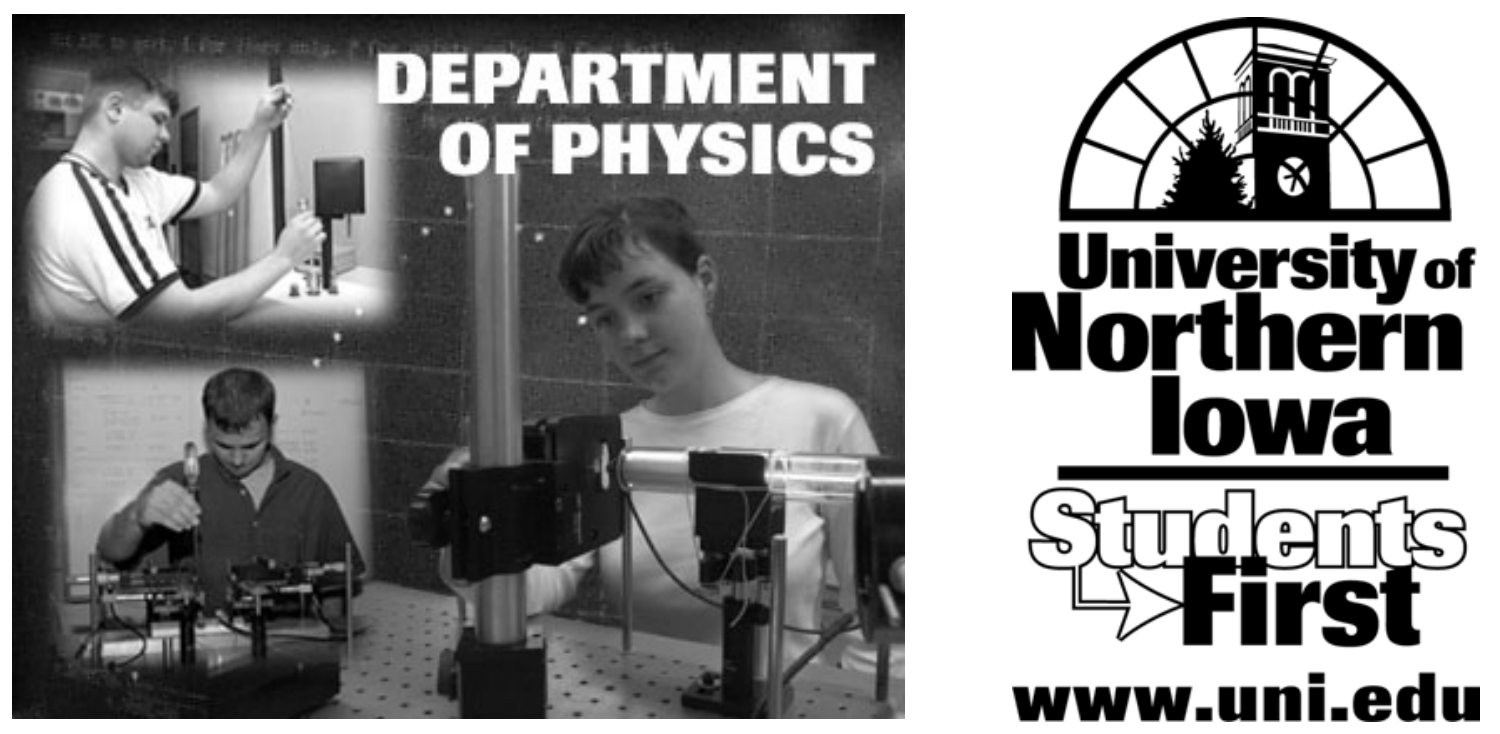
